\title{
The effect of hydrodynamic flow unsteadiness on heat transfer enhancement in a channel with square ribs
}

\author{
Nikolay Dushin", Nikolay Mikheev, Irek Davletshin, Anton Paereliy, Olga Dushina \\ Kazan Scientific Center of RAS, 420111, Kazan, Russia
}

\begin{abstract}
The heat transfer coefficient has been measured in the channel with square ribs for the case of periodic flow rate variation. New technique for heat transfer coefficient measurements has been applied in the study. It allows simultaneous surface heating and estimation of its local temperature. Two different patterns of heat transfer coefficient behavior in the gap between the ribs have been obtained. The first pattern corresponds to enhancement of both local heat transfer coefficient and average heat transfer if compared to its steady flow values. The second pattern exhibits considerable gain in heat transfer in the near-wake of the rib and its deterioration in other measurement regions. The reasons for such effects have been described on the basis of smoke visualization of flows.
\end{abstract}

\section{Introduction}

Currently, heat transfer enhancement in channels is based on turbulization of a thin nearwall region of flow, generation of large-scale vortices and boundary layer breakup. This approach allows significant enhancement of heat transfer accompanied by moderate increase in pressure drop. In this case, heat transfer augmentation is attributed to domination of turbulent transport over the molecular one and is governed by the linear scale of vortices and their velocity. Spanwise ribs mounted inside the channels are among the efficient ways to arrange such flows. Optimal rib pitch in the case of steady flow leads to a $26 \%$ increase in thermohydraulic performance in the Reynolds number range of $10^{4} \ldots 4 \times 10^{5}$.

Further augmentation of thermohydraulic performance can be achieved by variation of the rib shape, location of ribs and generation of flow rate pulsations [ $1-2]$. Thus, authors [2] obtained a $60 \%$ increase in heat transfer (if compared to the steady flow case) in pulsating flow through a single orifice. Besides, they mentioned that the maximum heat transfer coefficient shifted towards the orifice and documented five-fold increase in local heat transfer coefficient near the orifice. The shift of maximum heat transfer coefficient was also mentioned in [3] for the case of acoustic disturbances of different frequency and amplitude in the vicinity of a square rib, but the authors [3] did not achieve thermohydraulic performance comparable to [2]. Periodic blowing-suction of heat-transfer medium through the rib edge is another way to affect the flow pattern. Such an approach

* Corresponding author: ndushin@bk.ru 
has been thoroughly studied for the case of a backward-facing step flow [4 - 5]. These papers mainly focused on the control of flow pattern. In particular, the authors on average noted a 30\% reduction of the recirculation length. Heat transfer enhancement under periodic forcing of flow through the backward-facing step edge was studied in [6]. A 40\% increase in heat transfer coefficient in the near wake of the backward-facing step was obtained at the Strouhal number of 0.275 .

The review of research papers on hydrodynamics and heat transfer of separated pulsating flows shows that the majority of results have been obtained from numerical simulation. Meanwhile, experimental results are based mainly on the research of single obstacles in flows with some combinations of unsteadiness parameters. Thus, experimental research of thermohydraulic performance in configurations that are used in real-life engineering devices is a relevant task.

The present paper studies the effect of hydrodynamic unsteadiness of flow on heat transfer enhancement in a ribbed channel. Turbulent air flow in a $0.115 \times 0.15 \mathrm{~m}^{2}$ rectangular channel at the Reynolds number $\mathrm{Re}_{\mathrm{D}}=18200$ based on the hydraulic channel diameter was considered. Aluminum square spanwise ribs with the height $\mathrm{e}=13.5 \mathrm{~mm}$ were mounted in the channel. The rib-to-channel height ratio was $\mathrm{e} / \mathrm{H}=0.117$. The relative rib pitch was $\mathrm{p} / \mathrm{e}=10,15$, and 20. A smooth inlet section with $6: 1$ contraction was attached to the channel inlet. The latter was also equipped with a turbulence generating grid and an abrasive with a grit size of about $0.8 \mathrm{~mm}$. Measurements were carried out between the third and fourth ribs because of the attempt to reduce the measurement section length and exclude the possible effect of resonance in the experimental setup on measurement results.

\section{Experimental technique and procedure}

Local heat transfer coefficients were measured using a technique [7] that allowed simultaneous heating of the heat transfer surface and estimation of its local temperature from the measurement of corresponding electric resistance of a heating element. For this purpose, a composite epoxy plate was embedded into the test section wall. Dimensions of the plate were: length $450 \mathrm{~mm}$, width $200 \mathrm{~mm}$, thickness $1.5 \mathrm{~mm}$. The inwards facing surface of the plate was covered with a continuous zigzag copper strip. The strip width was $0.5 \mathrm{~mm}$. The strip spacing was $0.75 \mathrm{~mm}$ (the gap between adjacent parts of the strip was $0.25 \mathrm{~mm}$ ). The covered area was $420 \times 135 \mathrm{~mm}^{2}$. Heat transfer coefficients were measured for the regions $b_{1} \times B$ (measurement sections) equal to $9.5 \times 135 \mathrm{~mm}^{2}$ by the wires connected to the strip with a given spacing $(9.5 \mathrm{~mm})$.

To reduce the side heat losses, through grooves (sealed by a thin film) were made in the plate; and the area of wire surface was minimized. The reverse side of the plate was thermally insulated by two 10 -mm thick air chambers. External and dividing walls of the chambers were made of $9-\mu \mathrm{m}$ thick aluminum foil.

Local heat transfer coefficients were quantified using a constant heat flux method. Taking the heat loss into account, the heat transfer coefficient of the $i$ th measurement section of the plate was calculated from:

$$
\alpha_{i}=\frac{\frac{U_{i} U_{0}}{R_{0}}-\left[Q_{t i}+Q_{r_{i}}+Q_{s w_{i}}+Q_{x i}\right]}{S_{i} \Delta T},
$$

where $U_{0}$ and $R_{0}$ are the voltage drop and resistance of reference resistor, respectively; $U_{i}$ is the voltage drop at the $i$ th measurement section of the plate; $S_{i}$ is the area of the $i$ th measurement section of heat transfer surface.

Heat loss through thermal insulation was calculated for the $i$ th measurement section: 


$$
Q_{t i}=\alpha_{t i} \Delta T S_{i}, \frac{1}{\alpha_{t}}=\frac{\delta_{1}}{\lambda_{1}}+\frac{\delta_{t}}{\lambda_{t}}+\frac{1}{\alpha_{c}} .
$$

Here $\delta_{1}$ and $\lambda_{1}$ are the thickness and conductivity of the plate, respectively; $\delta_{t}$ and $\lambda_{t}$ are the insulation thickness and thermal conductivity coefficient of air, respectively; $\alpha_{c}$ is the coefficient of heat transfer from the plate surface.

Side heat losses, $Q_{s w i}$, through the plate were estimated by the formula derived for the straight ribs but transformed for the plate. Given that the temperature of heat-transfer medium is equal to the ambient air temperature, it is written as follows:

$$
Q_{s w}=\Delta T \sqrt{b_{1}^{2}\left(\alpha_{c}+\alpha_{t}\right)\left(\lambda_{1} \delta_{1}+\lambda_{2} \delta_{2} \frac{b_{2}}{b_{1}}\right)} \text { th } m L, \quad m=\sqrt{\frac{\alpha_{c}+\alpha_{t}}{\lambda_{1} \delta_{1}+\lambda_{2} \delta_{2} \frac{b_{2}}{b_{1}}}},
$$

where $b_{1}$ and $b_{2}$ are the pitch and width of the wires, respectively; $\delta_{1}$ and $\lambda_{1}$ are the thickness and conductivity of wires, respectively; $\Delta T$ is the temperature difference between the measurement sections of the plate and the heat-transfer medium:

$$
\Delta T=T_{w}-T_{f}=\frac{1}{K}\left(\frac{U_{i} R_{0}}{U_{0} R_{20_{i}}}-1\right)+20-T_{f} .
$$

In (4), $K$ is the temperature coefficient of the resistance thermometer; $R_{i}$ is the resistance of the measurement section strips at the temperature $T_{i} ; R_{i 20}$ is the nominal resistance of the measurement section strips at $20^{\circ} \mathrm{C}$.

Heat supplied to the $i$ th measurement section with the width $\Delta \mathrm{x}$ from adjacent sections was calculated from:

$$
Q_{x i}=-\lambda_{1} \delta_{1} B\left(\frac{T_{i-1}-2 T_{i}+T_{i+1}}{\Delta x}\right) .
$$

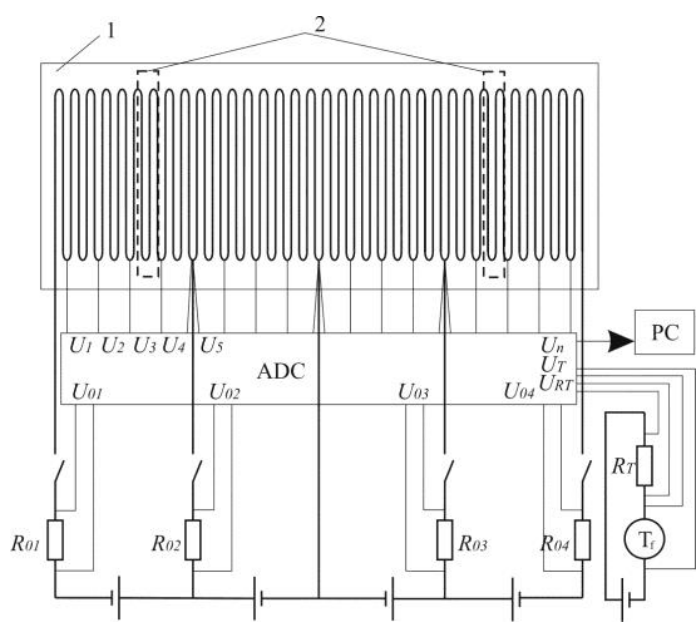

Fig.1. Schematic diagram of heat transfer coefficient measurement: 1 - heat transfer plate; 2 - location of ribs
Heat loss due to radiative heat transfer was not included in (1) due to its negligible value.

The plate was heated by an automotive battery. Ambient air temperature was measured by a platinum resistance thermometer Pt100. Voltage drop was measured by a 14-bit analog-to-digital converter Lcard E14-140M (resolution up to 19.5 $\mu \mathrm{V}$ ) connected to PC (fig. 1). Air flow rate was controlled by an ultrasonic flowmeter IRVIS-RS4Ultra. Flow rate fluctuations were generated by a shaped rotating flap mounted at the measurement section outlet. The rotation frequency was controlled by a frequency converter Vacon 10. 


\section{Results and discussion}

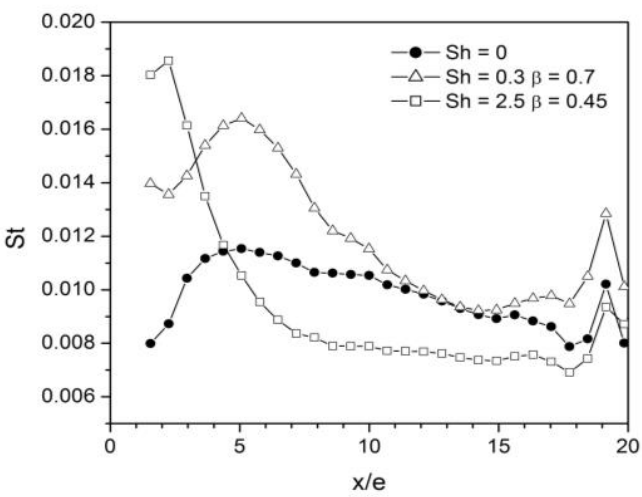

Fig.2. Local heat transfer between the ribs
Fig. 2 shows typical measurements of local heat transfer coefficient at the regimes with heat transfer enhancement and their comparison with the steady flow. Variation of frequency and amplitude of forced pulsations can result in different effects. For example, $44 \%$ heat transfer enhancement is observed in separation regions at $\mathrm{Sh}=0.3, \beta=\mathrm{A}_{\mathrm{u}} / \overline{\mathrm{u}}=0.7$ (here the Strouhal number, Sh, is based on the steady flow reattachment length). The integrated dimensionless heat transfer coefficient grows by $23 \%$. At the same time, the regime with $\mathrm{Sh}=$

$2.5, \beta=0.45$ is characterized by heat transfer deterioration over the largest part of the gap between the ribs. This results in reduction of the integrated dimensionless heat transfer coefficient by approximately $4 \%$, but significant heat transfer enhancement is observed in the rib near wake (2.12 times), and the peak heat transfer is shifted towards the rib.

To find the reasons for such different effects, the flow was visualized with smoke. The visualization in the first case demonstrated a large vortex that was formed behind the rib in the flow deceleration phase and shed downstream in the acceleration phase. The vortex diameter was 2-2.5 times the rib height. The second case exhibited a vortex with high angular velocity and the diameter comparable to the rib size. In the flow deceleration phase this vortex nestled against the rib and then was shed to the main flow. The air in the remaining part of the gap between the ribs behaved like a solid body almost without mixing with the mean flow.

\section{Conclusions}

New measurement technique has been employed to estimate the heat transfer coefficient in the flow with periodic flow rate pulsations in the channel with square ribs. Depending on the flow rate frequencies, different flow regimes can occur in the channel, including the ones with enhanced local heat transfer in the whole gap between the ribs and the ones with considerable heat transfer augmentation in the rib near wake and decrease over the remaining part of the gap. Smoke visualization of flow has revealed essential rearrangement of the flow pattern, which is responsible for the discovered effects.

\section{References}

1. G. Xie, S. Zheng, W. Zhang, B. Sunden, Applied Thermal Eng. 61, 289 (2013)

2. I. Davletshin, N. Mikheev, High Temp., 50212 (2012)

3. B. Cukurel, C. Selcan, M. Stratmann, Int. J. Heat Mass Transfer 91, 848 (2015)

4. S. Saric, S. Jakirlic, C. Tropea, Trans. ASME J. Fluids Eng. 127, 879 (2005)

5. A. Das Gupta, P. Zhao, S. Ray, 54th AIAA Aerospace sciences Meeting (2016)

6. G. Ree, H. Sung, Numerical Heat Transfer A, 37, 733 (2000)

7. N. Dushin, N. Mikheev, I. Gazizov, I. Davletshin, Russian Aeronautics (2017) (in press) 